\title{
Détermination du régime des bovins exploitant des parcours agropastoraux soudano-sahéliens (Sénégal): variations des teneurs en épidermes végétaux des fèces
}

\author{
MCB Diallo 1, H Guérin 1, V Freycon 2, D Richard ${ }^{3}$ \\ 1 IEMVT-CIRAD, service d'Alimentation, 10, rue Pierre-Curie, 94704 Maisons-Alfort Cedex; \\ 2 CTFT-CIRAD, 45 bis, avenue de la Belle-Gabrielle, 94736 Nogent-sur-Marne, France; \\ ${ }^{3}$ LNERV/ISRA, BP 2057, Dakar-Hann, Sénégal
}

\begin{abstract}
Summary - Determination of feed composition of cattle on Sudano-Sahelian pastures (Senegal) from the proportion of plant epiderms in the faeces. The proportions plants epiderms in faeces of cattle were found to vary according to sample preparation between animals in the same herd and between days. It is necessary to link results to a precise description of methods.
\end{abstract}

Des fèces de bovins ont été prélevées en saison des pluies pour déterminer les variations interindividuelles et journalières des choix alimentaires. Le but est de proposer des normes d'échantillonnage correspondant à un objectif de précision pour la description qualitative et quantitative des régimes.

Matériel et méthodes - Le troupeau étudié exploite des parcours agropastoraux hétérogènes en zone soudano-sahélienne au Sénégal $\left(13^{\circ} 45^{\prime}\right.$ de latitude nord et $15^{\circ} 40^{\prime}$ de longitude ouest). Des collectes individuelles de fèces sont effectuées sur les lieux de couchage et celles-ci sont conservées dans de l'alcool à $90^{\circ}$ (Diallo, 1990). Chaque échantillon est soumis à 2 techniques d'analyse histologique : l'analyse directe des refus de tamisage correspondant à $11 \%$ de la matière sèche fécale (MS), ou "méthode classique" (Baumgartner et Martin, 1939), et celle de la suspension de centrifugation ne représentant que $0,3 \%$ de la matière sèche fécale (Diallo, 1991). Un premier essai porte sur les fèces de 5 bovins, récoltées durant 5 j consécutifs pour étudier les variations de leur composition botanique en fonction du jour de récolte et de la méthode de préparation des lames. Une analyse de variance univariable a été utilisée sur les résultats individuels (Stat ITCF, 1988). Le deuxième essai de prélèvements est constitué de 26 échantillons individuels de fèces de bovins d'un même troupeau, mais d'âges et d'états physiologiques différents, ayant pâturé ensemble au cours d'une journée. Cet essai permet d'évaluer par analyse factorielle des correspondances (AFC), suivie d'une classification automatique, la ressemblance des régimes des bovins en fonction de leurs choix alimentaires. De plus, le nombre de bovins, $n$, à utiliser pour atteindre une précision relative, $d r$, désirée du nombre de fragments végétaux détectés, est calculé par la formule suivante (Stein, 1945) :

$$
n=\left(t_{(1-\alpha / 2)} \frac{V}{d r}\right)^{2}
$$

ou : $t_{(1-\alpha / 2)}:$ variable de student à $(n-1)$ ddl pour un risque $\alpha . V=$ coefficient de variation de la variable nombre de fragments végétaux détectés.

Résultats et discussion - Une cinquantaine de genres ou espèces végétales ont été identifiés, mais une trentaine d'entre eux apparaissent en "traces" avec une contribution spécifique présence (CSP : proportion en pourcentage du nombre total de fragments observés) inférieure ou égale à $0,6 \%$. L'essai 1 montre que le nombre moyen d'épidermes d'une plante donnée varie avec la méthode de préparation des lames (fig 1): les monocotylédones apparaissent plus nombreuses dans les préparations classiques que dans celles issues des suspensions de centrifugation, et inversement pour les dicotylédones, ce qui confirme nos travaux antérieurs (Diallo, 1990). Le jour de prélèvement influence aussi significativement la composition des fèces (fig 1), ce qui peut s'expliquer par les modifications des circuits du pâturage d'un 
jour à l'autre. L'essai 2 permet de distinguer, au sein du troupeau, plusieurs groupes d'animaux (tableau I), se distinguant par des régimes significativement différents. Notons que, suivant la méthode de préparation des lames, les groupes d'animaux mis en évidence ne sont pas identiques. Pour la plus grande partie des items végétaux, 15 bovins sont nécessaires pour obtenir une précision de $\pm 30 \%$ du nombre moyen de fragments détectés. En acceptant une précision de $\pm 45 \%$, cet effectif passe à 7 bovins. Ce nombre de bovins est à réajuster légèrement suivant la méthode de préparation des lames. La précision de $\pm 30 \%$ est admise dans la littérature (Holechek et Vavra, 1981) et celle de $\pm 45 \%$ serait acceptable dans notre milieu, considéré plus difficile que celui des auteurs cités.
En conclusion, l'analyse histologique des fèces peut fournir d'utiles informations qualitatives sur la composition botanique des régimes et ses variations journalières et individuelles. Quant à la détermination des quantités ingérées, les différences liées aux techniques de préparation des échantillons montrent qu'un important travail méthodologique reste à faire avant d'envisager des applications dans ces régions.

Baumgartner LL, Martin AC (1939) J Wild Management 3, 266-268

Diallo MCB (1990) Reprod Nutr Dev, Suppl 2, 211s-212s

Diallo MCB (1991) Thèse de doctorat, USTL Montpellier II (sciences et techniques du Languedoc), $262 \mathrm{p}$

Holechek JL, Vavra M (1981) J Range Manage 34, 337-338

Stein C (1945) Ann Math Stat 16, 243-258

Tableau I. Valeurs moyennes et extrêmes des contributions spécifiques présence (CSP en pourcentage des épidermes totaux) de quelques items végétaux suivant les groupes de bovins (G1 à G3, G'1 à G'3) constitutés par analyse factorielle des correspondances.

\begin{tabular}{|c|c|c|c|c|c|c|c|}
\hline \multicolumn{4}{|c|}{$\begin{array}{l}\text { Préparation classique } \\
\text {. }\end{array}$} & \multicolumn{4}{|c|}{ Suspension de centrifugation } \\
\hline $\begin{array}{l}\text { Groupes de bovins } \\
\text { Effectifs }\end{array}$ & $\begin{array}{c}G 1 \\
8\end{array}$ & $\begin{array}{l}\mathrm{G} 2 \\
2\end{array}$ & $\begin{array}{l}\text { G3 } \\
16\end{array}$ & $\begin{array}{l}\text { Groupes de bovins } \\
\text { Effectifs }\end{array}$ & $\begin{array}{l}G^{\prime} 1 \\
22\end{array}$ & $G_{3}^{\prime}$ & $G_{1}^{\prime \prime 3}$ \\
\hline Eragrostis sp (1) & 1 & 19 & 9 & Feretia apodenthera (2) & & & 8 \\
\hline Panicum laetum (1) & 0 à 3 & $18 \underset{4}{\text { à }} 19$ & $7 \underset{4}{7}+3$ & Indigofera sp (2) & $0 \underset{3}{\mathrm{a}} 4$ & 8 à 11 & $\begin{array}{l}8 \\
2\end{array}$ \\
\hline & & 3 à 5 & 2 à 9 & & 0 à 6 & 3 à 6 & 2 \\
\hline Sorghum arundin (1) & 7 & 3 & 3 & Tephrosia sp (2) & 15 & 3 & 8 \\
\hline Grewia sp (2) & 2 à 9 & $\begin{array}{l}6 \\
4 \text { à } 7\end{array}$ & $\begin{array}{c}4 \\
1 \text { à } 7\end{array}$ & & & & \\
\hline
\end{tabular}

1) monocotylédones; 2) dicotylédones.

a

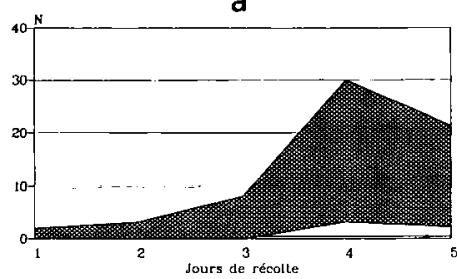

b

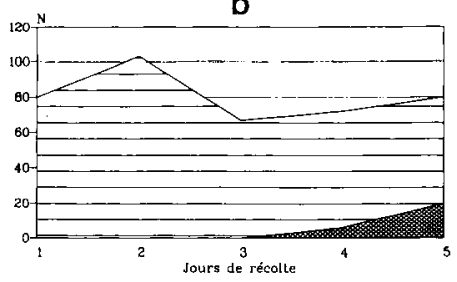

Fig 1. Exemples illustrant l'interaction des facteurs "jours de récolte des fèces de bovins et méthodes de préparation des lames-échantillons (500 fragments environ observés sur 5 lames)" en saison des pluies. Méthodes de préparation des lames : classique; $\square$ suspension. $N$ : nombre moyen de fragments par lame. a: monocotylédones Andropogon pseudapricus (exemple de graminés); b: dicotylédones Tephrosia sp (exemple de légumineuse). 